\title{
MANIFESTACIONES DE CULTURA EMPRESARIAL: UNA MIRADA DESDE LA ASOCIATIVIDAD EN LAS MIPYMES
}

\author{
Por: Laura Marcela López Posada ${ }^{1}$
}

\section{RESUMEN}

Este artículo analiza las manifestaciones de identificación cultural de nueve grupos asociativos denominados "Programas de Desarrollo Empresarial Sectorial" (PRODES) Colombianos estudiados en Antioquia, Bogotá, Tolima y Caldas; compuesto por 5, 2, 1 y 1 Prodes respectivamente. Los Prodes se estudiaron a través la Asociación Colombiana de Pequeñas Industrias (ACOPI), institución líder en el desarrollo, promoción y consolidación de la estrategia de asociatividad Prodes en Colombia. Esta investigación cualitativa desarrollo 60 entrevistas en profundidad, tres historias de vida, dos focus group y la observación como técnica de investigación transversal al desarrollo del trabajo de campo. Los resultados del estudio demuestran que los estereotipos regionales, las prácticas (símbolos, héroes y rituales) y los valores compartidos son manifestaciones de identificación cultural y de pertenencia a un Prodes, independientemente si se han creado o no relaciones sociales internas fuertes entre integrantes del mismo.

Palabras clave: Asociatividad, Prodes, Cultura Empresarial, Mipymes.

JEL: Z13, M14.

1. PhD. en Emprendimiento, Cooperativismo e Innovación por Mondragon Unibertsitatea (País Vasco). Universidad del Tolima, Facultad de Ciencias Económicas y Administrativas. Correo electrónico: lmlopezpo@ut.edu.co

Artículo recibido: 13 de marzo de 2015. Aprobación definitiva: 12 de mayo de 2015. 


\title{
MANIFESTATIONS OF CORPORATE CULTURE: A VIEW FROM THE ASSOCIATIVITY IN SMSES
}

\author{
By: Laura Marcela López Posada
}

\begin{abstract}
This article analyzes the manifestations of cultural identification in nine associative groups called Colombian Sectoral Enterprise Development Programs (Prodes) studied in Antioquia, Bogotá, Tolima and Caldas; compound by 5,2,1 and 1 Prodes respectively. The Prodes were studied through of The Colombian Guild of Small Industries (Acopi), which is a leader in the development, promotion and consolidation of associativity Prodes strategy in Colombia. This qualitative research included sixty interviews, three stories of life, two focus group and the observation as a research technique transverse to the development of fieldwork. The study results show that regional stereotypes, practices (symbols, heroes and rituals) and the shared values are manifestations of cultural identification and belonging to a Prodes, regardless if they have been created or not internal strong social relationships among theirs members.
\end{abstract}

Keywords: Associativity, Prodes, Corporate Culture, SMSES.

JEL: Z13, M14. 


\title{
MANIFESTAÇÃO DA CULTURA EMPRESARIAL: UM OLHAR DESDE A ASSOCIAÇÃO NAS PME
}

\author{
Por: Laura Marcela López Posada
}

\section{RESUMO}

Este artigo analisa as manifestações de identificação cultural de nove grupos em parceria chamados "Programas de Desenvolvimento de Negócios do Setor" (PRODES) Colombianos. Esses PRODES foram avaliados pela Associação Colombiana de Pequenas Indústrias (ACOPI) nos estados de Antioquia, Bogotá, Tolima e Caldas, conformado de 5, 2, 1 e 1, respetivamente. ACOPI é líder em desenvolvimento, promoção e consolidação da estratégia de parceria na instituição Colômbia Prodes. Esta pesquisa qualitativa desenvolveu 60 entrevistas, três histórias de vida, dois grupos focais e observação como técnica de pesquisa transversal ao trabalho de campo. Os resultados do estudo mostram que estereótipos regionais, práticas (símbolos, heróis e rituais) e valores compartilhados são manifestações de identidade cultural e de pertença a um Prodes, independente de tiver ou não fortes relações sociais internas entre membros do mesmo.

Palavras-chave: Parceria, Prodes, cultura empresarial, PME.

JEL: Z13, M14. 


\section{INTRODUCCIÓN}

El presente artículo busca analizar las manifestaciones de identificación cultural de nueve Prodes Colombianos estudiados en Antioquia, Bogotá, Tolima y Caldas. Estas manifestaciones de identificación cultural son estudiadas a través de los estereotipos regionales, las prácticas (símbolos, héroes y rituales) y los valores compartidos de los grupos asociativos objeto de estudio en el presente artículo, los Prodes. El artículo se desarrolla en cuatro partes: justificación y revisión de antecedentes teóricos y conceptuales desarrollados en la introducción, metodología, presentación de resultados y de discusión y conclusiones.

La investigación tiene motivos prácticos y motivos científicos que la justifican. Entre los motivos prácticos se destaca que el estudio de la estrategia de asociatividad Prodes responde a un problema de coyuntura nacional colombiana, dado que la estructura empresarial del país está representada en su mayoría por micro y pequeñas empresas que tienen graves problemas de competitividad. Se resalta que de 1.341 .368 empresas en Colombia, el 96,4\% de los establecimientos son micro empresas, mientras que otro $3,5 \%$ son pequeñas y medianas y un $0,1 \%$ son grandes (Congreso de Colombia, Ley 590 de 2000). Por lo tanto, se estudian las Mipymes no sólo porque conforman la mayor parte de estructura empresarial del mercado colombiano, sino porque en ellas se focalizan los principales problemas de competitividad empresarial colombiana.

Otra motivación práctica de realizar ésta investigación tiene que ver con la oportunidad de divulgar la experiencia de asociatividad colombiana Prodes, no sólo en el contexto nacional colombiano, sino también en el internacional. En Colombia el Prodes es una estrategia de asociatividad liderada por Acopi y diseñada para mejorar la competitividad de las Mipymes. Sin embargo, no todas las seccionales de Acopi conocen las ventajas del Prodes, y no todas lideran esta estrategia de asociatividad con sus afiliados. Se espera que los resultados de la presente investigación sirvan como insumo para la toma de decisiones y planeación estratégica de instituciones de apoyo gubernamentales y no gubernamentales, como: Acopi, Cámara de Comercio, Sena, Gobiernos Locales en asuntos relacionados con el diseño de estrategias de asociatividad, y su implementación en diferentes zonas del país. Además, permitirá a las autoridades nacionales y regionales hacer ajustes a las agendas de competitividad Nacional y Regional, tomando los resultados de la investigación como un diagnóstico de la estrategia de asociatividad Prodes de algunas regiones de Colombia. Este diagnóstico puede servir para elaborar y reelabora planes de desarrollo de acuerdo a las necesidades y a los intereses de las Mipymes del Estado colombiano.

Entre los motivos científicos se resalta la oportunidad de estudiar las manifestaciones de cultura empresarial a través la dimensión cultural individualismo y colectivismo (Triandis, 1994, 1995; Hofstede, 1999), perspectiva teórica poco estudiada en el contexto colombiano. 


\section{LA ASOCIATIVIDAD EN LAS MIPYMES: LOS PRODES}

La asociatividad empresarial tiene por objeto principal mejorar la gestión, la productividad y competitividad de las empresas asociadas (Pallares, 2004), y en países como Colombia surge la mayoría de veces como mecanismo de cooperación entre las micro, pequeñas y medianas empresas para sobrevivir (Iguera, 2006) en el mercado local. Los procesos de asociatividad son ejercicios voluntarios y sin remuneración, son esfuerzos conjuntos con otros participantes para lograr un objetivo común, manteniendo su independencia jurídica y autonomía gerencial (MCIT, 2005; Iguera, 2006; Arcila, 2006; Aguirre y Pinto, 2006; Vega, 2008); pero fundamentando las relaciones en la cooperación, la confianza mutua y la reciprocidad.

Un concepto reconocido en el tema de asociatividad en las Mipymes es el presentado por Rosales, y la define como “(...) un mecanismo de cooperación entre empresas micro, pequeñas y medianas, en donde cada empresa participante, manteniendo su independencia jurídica y autonomía gerencial, decide voluntariamente participar en un esfuerzo conjunto con los otros participantes para la búsqueda de un objetivo común” (1997: 97).

El objeto de estudio del presente artículo son los Programas de Desarrollo Estratégico Sectorial (Prodes). Un Prodes se define como un conjunto de actividades asociativas desarrolladas en las micros, pequeñas y medianas empresas, orientadas al mejoramiento de la gestión, la productividad y la competitividad de las mismas, en el corto, mediano y largo plazo (Pallares, 1999, 2004; Acopi, 2001; Ortiz, 2002: 2). Acopi plantea que el Prodes “(...) es un espacio de aprendizaje empresarial basado en el intercambio de experiencias y saberes gerenciales, orientado hacia la generación de una nueva visión colectiva, integral, asociativa y estratégica, para enfrentar competitivamente la nueva etapa de la internacionalización de las economías" (Acopi, 2001: 2).

Los Prodes son una red inter-organizacional, pero también una forma de asociatividad. Los Prodes se relacionan con una red inter-organizacional, porque en ellas se construyen relaciones de cooperación de largo plazo entre organizaciones, consumidores, proveedores, competidores, y otros actores con los que se mantiene el control sobre sus propios recursos, pero en conjunto se decide sobre su uso (Ebers, 1997). En estas formas de asociatividad, por lo general los problemas se resuelven a través de concertación de reglas y normas de reciprocidad que aseguran la cooperación (Powell, 1990; Uzzi, 1997). Lo importante de la definición de asociatividad es que pone de relieve el tema de la reciprocidad; se espera que la cooperación sea mutua y recurrente entre los actores que participan en la red (Mideplan, 2002; Aguirre y Pinto, 2006), en este caso del grupo asociativo Prodes.

Los Prodes nacen, bajo el lema "cooperar para competir", dirigido por un sector gremial específico, en este caso Acopi, orientado hacia la generación de una nueva visión colectiva, integral, asociativa y estratégica, para enfrentar competitivamente la nueva etapa de la internacionalización de la economía (Cogollo, León y Vergara, 2005: 35-36).

En general, la estrategia de asociatividad Prodes se consolida en las Mipymes colombianas como una alternativa para generar competitividad empresarial y 
desarrollo local. Las entidades gremiales, como Acopi, son actores e instituciones claves en el desarrollo de dicho Programa en las regiones de Colombia. Las ventajas que obtienen las empresas al implementar estrategias de asociatividad, tales como clúster y Prodes son variadas, y van desde competitividad en el entorno económico nacional e internacional, hasta relaciones de amistad y colaboración perdurables en el tiempo.

\section{SOBRE EL CONCEPTO DE CULTURA}

En el presente artículo se evidencia un interés por estudiar la cultura de un grupo social, en éste caso de Prodes regionales colombianos. El interés no es estudiar la cultura de los individuos, ni de las organizaciones por separado, sino de los grupos de empresas que conforman los Prodes objeto de estudio. Las investigaciones que se encuadran en este enfoque, el de cultura de grupo o subgrupos, intentan describir el objetivo a que sirven y las funciones que desempeñan las creencias y símbolos en las organizaciones, presentándolos ambos como elementos necesarios para el orden y la estabilidad social (Evered, 1983; Ott, 1989). Parte de estos trabajos se centran en analizar la forma en que la cultura puede servir como elemento integrador de los individuos en la organización o como instrumento de control interno (Wilkins \& Ouchi, 1983).

La cultura es un tema de interés multidisciplinar, sobre el cual han escrito sociólogos, psicólogos, antropólogos, filósofos, administradores, ingenieros; cada disciplina tiene una motivación diferente para ahondar en temáticas relacionadas con la cultura. Una pregunta que sería pertinente hacer es, por qué se estudia la cultura, es acaso la cultura un tema transversal a la realidad del hombre y la sociedad. La cultura de acuerdo con el proyecto $\mathrm{GLOBE}^{2}$ es definida como (...) los motivos compartidos, valores, creencias, identidades e interacción de eventos significativos que resultan de experiencias comunes de los miembros de las colectividades, y las cuales son transmitidas de generación en generación (House, Hanges, Javidan, Dorfman \& Gupta, 2004: 15; Ogliastri, Mcmillen, Altschul, Arias, Bustamante, Dávila, Dorfman, Dela Coletta, Fimmen, Ickis, y Martínez, 1999: 30-33).

Es claro para autores como Gelfand, Erez \& Aycan (2007) y House et al. (2004) que existen múltiples definiciones de cultura, se ha dicho que (...) así como no existe una definición unívoca de liderazgo, tampoco la hay de cultura. Generalmente, el término cultura es usado por los científicos sociales para referirse a una serie de parámetros establecidos de la colectividad, que diferencia a cada una de la otra de una forma significativa e intrínseca (House et al., 2004: 16). Gelfand et al (2007: 481) reúne algunas definiciones de cultura, como: La cultura ha sido definida como el ser humano que hace parte del ambiente (Herkovits, 1955), incluyendo elementos tanto objetivos como subjetivos (Triandis, 1972); como un juego de refuerzos

2. Globe: Global leadership and Organizational Effectiveness Research Program. GLOBE es un proyecto mundial, multimetódico, multifacético, diseñado para responder preguntas relacionadas con comportamiento y el liderazgo organizacional efectivo en las sociedades (influencia de la cultura en el liderazgo y el comportamiento organizacional). Este concepto fue concebido en el verano de 1991, y fue materializado con su fundación en octubre de 1993, y a partir de ese momento, empezó la recolección de datos por 62 sociedades (House \& Javidan, 2004: 11). 
(Skinner, 1981); como la programación colectiva de la mente (Hofstede, 1999); como un sistema de significados compartidos (Shweder \& Levine, 1984); como todos los modos de vida históricamente creados, explícitos como implícitos, racionales y no racionales, que existen en cualquier tiempo determinado como guías potenciales del comportamiento de los hombres (Kluckhohn, 1954); o como los procedimientos habituales o las maneras de hacer las cosas (Triandis, 1994); también se ha definido como (...) las formas, hábitos y valores culturales de conducta y acción, asumidos y re-definidos a partir del individuo y su relación con el campo social del y para el equipo de trabajo en el cual interactúan (Llanque, 2003: 1587).

House et al. (2004: VI) explican que el proyecto de investigación GLOBE, examina la cultura como prácticas y valores. Las prácticas son los actos o (...) la forma como se hacen las cosas en esa cultura, y los valores son los artefactos debido a que son creados por el humano, y en este caso específico, son juicios sobre la forma en que deben hacerse las cosas.

Hablar de cultura en la empresa o en un grupo de empresas implica relacionar prioritariamente el conjunto de valores, necesidades y expectativas, creencias, políticas y normas generadas, aceptadas y practicadas, históricamente por los actores y agentes de la organización. Hofstede (1999: 34) señala que la cultura puede ser vista como un software mental ${ }^{3}$, que distingue a los miembros de una organización de la otra. Vale la pena mencionar que los antropólogos sociales se refieren a la cultura, como "cultura dos". La cultura (dos) es siempre un fenómeno colectivo porque es compartido, al menos parcialmente, por las personas que viven o han vivido dentro del mismo entorno social, en el cual la han aprendido.

Dadas las diferentes definiciones que se ofrecen en las ciencias sociales sobre la noción de cultura, y especialmente cultura de la organización, no se puede más que reconocer (DiMaggio, 1994) que se trata de un término resbaladizo y ambiguo. Aunque el concepto de cultura de la organización está infrautilizado, algunos autores lo encuentran bastante útil para entender el comportamiento tanto de la organización como de sus miembros (Schein, 1985, 1996; Aguirre y Rodríguez, 1995)

Aunque las definiciones de la cultura varían de un autor a otro, se enfatiza en que la cultura es compartida, es adaptable en tiempo, en espacio y en generaciones. La cultura no es manipulable, se construye históricamente. Por lo tanto, el ser humano a través de su interacción con la sociedad adscribe nuevas prácticas, hábitos, ritos, símbolos, normas y valores que permiten configurar una cultura particular y diferente frente a otras (Andrews, Basler y Coller, 1997).

\section{a. Diferencias entre cultura nacional y cultura organizacional}

Al tratar de conceptualizar cultura se presentan dificultades, dado que no existe un único concepto de cultura, y las características de la cultura nacional o regional, no son las mismas que la denominada cultura organizacional. Hofstede

3. El origen de nuestros programas mentales está en los entornos sociales en los que hemos crecido y acumulado nuestras experiencias vitales. Los programas mentales difieren tanto como los entornos sociales en los que se han adquirido (Hofstede, 1999: 34). 
(1999: 295) afirma que se suele aplicar la palabra cultura tanto a naciones como a organizaciones, pensando que se trata de fenómenos idénticos, y no es así (...) una nación no es una organización y los dos tipos de cultura son de índole diferente. La diferencia entre cultura nacional y cultura organizativa se debe a los distintos papeles que desempeñan en cada de las manifestaciones de cultura, como son: símbolos, héroes, rituales y valores -reagrupados los tres primeros bajo el nombre de "prácticas"-.

La cultura nacional, así como la cultura regional permea la cultura de los individuos dentro de las organizaciones y dentro de un grupo social determinado. Las culturas regionales explican las diferencias dentro de los países. Hofstede (1983: 75) afirma que la cultura nacional y regional es importante para la gestión de las organizaciones o de grupos de empresas por tres razones. La primera, es política. Las naciones son unidades políticas, arraigadas en historia, con sus propias instituciones: de sistemas de asociación, de las formas de gobierno, de los sistemas legislativos, de los sistemas educativos, del trabajo. La segunda razón, por la que la nacionalidad es importante es sociológica. La nacionalidad o la regionalidad tienen un valor simbólico en los ciudadanos. Este autor afirma que parte de la identidad se deriva del valor simbólico del hecho de pertenecer a una nación o a una región. Las diferencias nacionales y regionales son sentidas por la gente como parte de su realidad y de su identidad; y por lo tanto, se adscriben a espacios de socialización, como la empresa y el escenario empresarial. La tercera razón por la que la nacionalidad es importante es psicológica. Hofstede (1983) señala que el pensamiento es condicionado en parte por características de la cultura nacional y regional.

La nacionalidad o la regionalidad tienen un valor simbólico para los ciudadanos de una región, se asigna una valoración positiva o negativa al hecho de pertenecer a determinada nación o región, y las diferencias en atributos regionales son sentidas por la gente como parte de su realidad y de su identidad. Por ejemplo, los estereotipos regionales pueden afectar las acciones y comportamientos de un grupo social. Un estereotipo es la generalización simplificada socialmente compartida sobre las personas que pertenecen a un grupo particular (Forsyth, 2006; Fernández, 2011), y se fundamenta en creencias populares sobre los atributos que caracterizan a una categoría social y sobre los que hay un acuerdo sustancial (Mackie, 1973: 435).

Los estereotipos se asignan a grupos de gentes en forma de etiquetas verbales (Vinacke, 1956), provocando una simplificación o una orientación selectiva de las percepciones individuales que podrían conducir a distorsiones más o menos graves de la realidad objetiva. Las personas pueden darle un valor simbólico a dichos estereotipos regionales (positivos o negativos), y estos pueden ser difundidos "irresponsablemente" como atributos de identificación regional; dichos estereotipos no siempre se fundan en experiencias directas vividas, sino que pueden ser simplemente una reproducción de ideas y creencias preconcebidas falsas e inexactas sobre la realidad (Andrade de Souza, 2005; Fernández, 2011). 
Cabe señalar, que a nivel nacional o regional las diferencias culturales pueden observarse principalmente en los valores y en las prácticas, menos en simples estereotipos regionales. A nivel organizativo, las diferencias culturales residen sobre todo en las prácticas y menos en los valores. Las prácticas organizativas, por el contrario, se aprenden a través de la socialización en el lugar de trabajo, al que la mayoría de las personas llegan con el grueso de sus valores ya firmemente arraigados (Hofstede, 1999: 297 - 298).

En general, las culturas organizativas son un fenómeno en sí mismas, diferentes en muchos aspectos de las culturas nacionales. Una organización es un sistema social de índole diferente a una nación, aunque sólo sea porque los miembros de la organización han tenido habitualmente cierta influencia en su decisión de unirse a ella, participan en ella sólo durante las horas de trabajo y pueden un día abandonarla. Los resultados de las investigaciones relacionadas sobre culturas nacionales y sus dimensiones han demostrado ser útiles para comprender las culturas organizativas.

\section{METODOLOGÍA}

La presente artículo logra identificar las manifestaciones de la cultura empresarial en algunos Prodes de Antioquia, Bogotá, Tolima y Caldas. Es un estudio de carácter cualitativo, y busca investigar la realidad de los Prodes objeto de estudio, no desde su microestructura ${ }^{4}$, sino desde su macroestructura ${ }^{5}$. Los sujetos objeto de estudio de la presente investigación son los que aportan información del Prodes como un todo, y son: los directivos de Acopi que lideran la estrategia Prodes, y los empresarios que integran cada uno de los grupos asociativos sujetos a estudio.

Se utiliza la combinación de diferentes técnicas de investigación cualitativa garantizando la triangulación de datos y de informantes, así: las entrevistas en profundidad, las historias de vida, la observación, el focus groups y la revisión documental con empresarios de Mipymes afiliados a Acopi, y que participan o participaron en la estrategia de asociatividad, Prodes. La combinación de técnicas de investigación, permite identificar los puntos de vista de cada uno de los actores involucrados en el presente estudio, así: Directores ejecutivos de Acopi, fundadores y promotores de los Prodes en Colombia, Directivos de Mipymes que participan de los Prodes regionales.

Se realizaron aproximadamente 60 entrevistas en profundidad semiestructuradas en nueve (9) Prodes heterogéneos de cuatro regiones de Colombia (Antioquia, Tolima, Bogotá y Caldas) (Ver Tabla 1. Entrevistas Prodes regionales -Colombia y Tabla 2. Prodes Objeto de Estudio).

4. Estructura que forma parte de otra estructura más amplia.

5. Estructura o sistema máximo que engloba otros menores e interrelacionados. 
Tabla 1. Entrevistas Prodes regionales (Colombia)

\begin{tabular}{|l|c|c|c|c|c|c|c|}
\hline Regiones & $\begin{array}{c}\mathbf{N}^{\circ} \text { de } \\
\text { Prodes } \mathbf{x} \\
\text { Región }\end{array}$ & $\begin{array}{c}\mathbf{N}^{\circ} \text { de } \\
\text { Prodes para } \\
\text { el dllo. de } \\
\text { campo }\end{array}$ & $\begin{array}{c}\text { Entrevistas } \\
\text { directivos y } \\
\text { ex-directi- } \\
\text { vos Acopi }\end{array}$ & $\begin{array}{c}\text { Entrevistas } \\
\text { de Historia } \\
\text { de Vida }\end{array}$ & $\begin{array}{c}\text { Entrevistas } \\
\text { a directivos } \\
\text { de otras } \\
\text { entidades } \\
\text { de apoyo a } \\
\text { la Mipyme } \\
\text { colombiana }\end{array}$ & $\begin{array}{c}\text { Entrevistas } \\
\text { a gerentes } \\
\text { de Mipymes } \\
\text { por Prodes }\end{array}$ & $\begin{array}{c}\text { Total de } \\
\text { entrevistas } \\
\text { en profun- } \\
\text { didad por } \\
\text { Región }\end{array}$ \\
\hline Antioquia & 44 & 5 & 4 & 1 & 0 & 22 & 27 \\
\hline Tolima & 26 & 2 & 3 & 0 & 1 & 11 & 15 \\
\hline Bogotá & 1 & 1 & 1 & 2 & 2 & 6 & 11 \\
\hline Caldas & 0 & 1 & 2 & 0 & 0 & 5 & 7 \\
\hline Total & 48 & 9 & 10 & 3 & 3 & 44 & 60 \\
\hline
\end{tabular}

Fuente: Autor, 2013.

Tabla 2. Prodes objeto de estudio

\begin{tabular}{|c|c|c|c|c|}
\hline Nombre del Prodes & Departamento & No Prodes x Dpto. & $\begin{array}{l}\text { Año de } \\
\text { creación }\end{array}$ & Supervivencia \\
\hline Metalmecánico Intec & Antioquia & \multirow{5}{*}{$\begin{array}{l}5 \text { Prodes de } \\
\text { Antioquia }\end{array}$} & 2003 & Supervivencia \\
\hline Ropa Infantil (Pro-Bebé) & Antioquia & & 2004 & Supervivencia \\
\hline Comunicación Gráfica & Antioquia & & 2005 & Supervivencia \\
\hline Vía Primavera & Antioquia & & 2009 & Supervivencia \\
\hline Energía & Antioquia & & 2009 & Supervivencia \\
\hline Confección & Tolima & \multirow{2}{*}{2 Prodes de Tolima } & 2008 & Supervivencia \\
\hline Tamal-Lechona & Tolima & & 2008 & Supervivencia \\
\hline $\begin{array}{l}\text { Tecnologías de informa- } \\
\text { ción (Tics) }\end{array}$ & Bogotá & $\begin{array}{l}1 \text { Prodes de } \\
\text { Bogotá }\end{array}$ & 2010 & $\begin{array}{c}\text { No } \\
\text { Supervivencia }\end{array}$ \\
\hline Metalmecánica & Caldas & $\begin{array}{l}1 \text { Prodes de } \\
\text { Manizales }\end{array}$ & $\begin{array}{l}\text { Desapa- } \\
\text { reció }\end{array}$ & $\begin{array}{c}\text { No } \\
\text { Supervivencia }\end{array}$ \\
\hline
\end{tabular}

Fuente: Autor, 2013.

La técnica de historia de vida se realizó con fundadores y promotores de los Prodes en Colombia, así: Zoilo Pallares Villegas, Jairo Díaz Valencia, y Juan Bautista Franco. La técnica de historia de vida permitió conocer y reconocer, aunque parcialmente, algunas de las características de identificación cultural construidas a través de la estrategia de asociatividad Prodes en algunas regiones de Colombia, específicamente las que son objeto de investigación. El focus group es una herramienta complementaria a las entrevistas en profundidad e historias de vida, $\mathrm{y}$ permitió identificar acuerdos, desacuerdos y contradicciones de los informantes en relación con los temas de estudio. Se realizan dos focus group, en dos de las 
seccionales de Acopi objeto de estudio que actualmente tiene Prodes vigentes $o$ activos, uno en Antioquia y otro en Bogotá.

Dado que las técnicas de recopilación de información, tales como: entrevistas en profundidad, historias de vida y focus group se realiza en el contexto laboral de los sujetos y organizaciones objeto de estudio, se aprovecha dicho espacio para aplicar la técnica de observación, la cual se materializó a través de los diarios de campo en el que se registraron las observaciones y las reacciones del investigador durante el ejercicio de su actividad (dificultades, observaciones, informantes, bloqueos, problemas, redefiniciones, e incluso sorpresas en el proceso investigativo). Se solicita información documental sobre Cultura del Prodes a las diferentes seccionales de Acopi incluidas en el estudio. (Antioquía, Bogotá, Caldas y Tolima).

Para el análisis de contenido de esta información se definieron unas categorías de análisis ${ }^{6}$, y con base en ellas se realizó al análisis de contenido de toda la información recopilada. Para la etapa inicial de codificación y clasificación de información de campo se uso el software de análisis de contenido Atlas-ti; posteriormente el investigador tuvo que volver sobre el documento de análisis para corroborar la eficacia de la codificación y clasificación de dicha información ${ }^{7}$.

\section{RESULTADOS Y DISCUSIÓN}

\section{a. Estereotipos regionales en los Prodes: mitos y realidades}

En las regiones de Antioquia, Tolima, Bogotá y Caldas se han configurado unos estereotipos regionales que les permite diferenciarse entre sí. Los grupos humanos de una región o territorio son los que dan significados, y valorizan el espacio, creando representaciones de identificación regional para diferenciarse de otros grupos (Arcila, 2006: 42). En este sentido, los estereotipos comportarían la asignación de ciertos rasgos a los individuos que son miembros de un grupo y también la atribución de ciertas diferencias respecto a los miembros de otros grupos. A continuación se hace un análisis de los estereotipos de las regiones de Antioquia, Tolima, Bogotá y Caldas -en su orden-, teniendo en cuenta alguna literatura escrita sobre el tema, y tomando en consideración el discurso subjetivo de empresarios entrevistados en el trabajo de campo, acerca de los rasgos de identificación de dichas regiones.

A continuación se resaltan algunos de los estereotipos regionales de Antioquia, Tolima, Bogotá y Caldas. Al contrastar, la información encontrada en la revisión de la literatura con la referenciada por los entrevistados de dichas regiones, se encuentran algunas correspondencias sobre sus calificativos. La región antioqueña ha sido históricamente valorada y reconocida por un desarrollo empresarial difícilmente

6. De acuerdo a los objetivos formulados se consideraron siete (6) categorías de análisis para explicar las manifestaciones de identificación cultural en los Prodes, así: (1) Prácticas, (2) Símbolos, (3) Héroes, (4)Rituales, (5)Tabúes, y (6) Estereotipos Regionales. Cada una de estas categorías tiene a su vez un conjunto de subcategorías de análisis que la soportan.

7. En la clasificación e interpretación de información de campo se empleó la categorización léxica, donde se da una clasificación de palabras según sus sentidos, con emparejamiento de sinónimos y de sentidos próximos; es decir, se analiza el contexto y los elementos vecinos al actual, y se escoge el significado que mejor encaja (Valles, 1999). 
comparable con otras regiones de Colombia. Entre los rasgos más representativos del antioqueño se resaltan: la motivación y voluntad para impulsar proyectos empresariales, la pujanza y tenacidad, el espíritu emprendedor y la capacidad para trabajar en equipo. Al examinar las causas que pudieron influir en la concepción de estos estereotipos regionales tan marcados como los observados en la región de Antioquia, se puede concluir que no hay una única causa ni geográfica, ni social, ni cultural para explicar el arraigo de estas prácticas y valores en dicha región. Es la suma de cada uno de los argumentos expuestos, los que valorizan el estereotipo antioqueño, con sus historias, mitos y realidades.

Lo mismo sucede en las regiones de Tolima, Bogotá y Caldas. No se puede atribuir a un sólo acontecimiento o situación los rasgos de identificación regional. Es más bien la suma de acontecimientos y vivencias las que a través del tiempo configuran un prototipo de identificación regional, que se transmite y se transforma de generación en generación. El tolimense ha sido estereotipado mayoritariamente porque sus habitantes se perciben como indiferentes, conformistas, egoístas y desconfiados, pero también como buenos trabajadores. Por otro lado, en la región de Bogotá el estereotipo está relacionado con gente tradicionalista, egoísta, desconfiada e individualista; pero también con potencial en el desarrollo político, educativo y cultural. Por último, el estereotipo caldense también se reconoce y se referencia como individualista, desconfiado, cerrado y con poca habilidad para trabajar en equipo; además, de cualidades de emprendimiento y arraigo regional. Parece ser que los estereotipos asignados a regiones como Tolima, Bogotá y Caldas no favorecen el trabajo asociativo, teniendo en cuenta que algunos rasgos de identificación común están relacionados con comportamientos tendientes al individualismo y a la desconfianza para trabajar con otros.

Los estereotipos regionales son percepciones y creencias sobre las cualidades que identifican a una región, son parte del sentido común y de las representaciones sociales que se hacen sobre un grupo social determinado. Las percepciones que tienen los entrevistados de Antioquia, Tolima, Bogotá y Caldas sobre las cualidades que las gentes de su región son tanto positivas como negativas; pero cuando se realiza una valoración negativa de un grupo con base al estereotipo, el resultado es el prejuicio ${ }^{8}$.

\section{b. Las prácticas colectivas en los Prodes: una forma de identificación colectiva}

Las prácticas son acciones que ayudan a influir comportamientos, según Hofstede (1999) las prácticas se pueden manifestar a través de los símbolos, los héroes o rituales. En los nueve Prodes estudiados se identificaron algunos símbolos generales de la estrategia Prodes, que tienen valor sólo para los empresarios que han participado o participaron en algún momento de dicha estrategia de asociatividad, así: Los Profos de Chile, punto de referencia para crear los Prodes en Colombia;

8. El prejuicio es un juicio anticipado, una opinión, una apreciación, que se hace antes de cualquier información o conocimiento más profundo, son sentencias que no cuentan con ningún juzgamiento reflexivo elaborado. Los prejuicios son opiniones livianas y arbitrarias, que repetidas innumerables veces figuran como verdades incuestionables (Andrade de Souza, 2005: 5). 
Acopi, entidad gremial creadora y promotora regional de la estrategia de asociatividad Prodes; Acopi Antioquia, seccional de Acopi con liderazgo, reconocimiento y experiencia en la creación, consolidación y desarrollo de Prodes en Antioquia; y los gansos, como imagen institucional de los Prodes a nivel nacional.

En los Prodes Vía Primavera (Antioquia), Tamal-Lechona (Ibagué) y Confección (Ibagué) también se identifican algunos símbolos representativos de los grupos. En el Prodes Vía Primavera (Antioquia) se encuentra el “Árbol Vía Primavera”, que es la imagen institucional de la Corporación Vía Primavera, y representa la cuadra "Vía Primavera", lugar arborizado donde se localizan cada uno de los empresarios miembros de dicho Prodes. Un lugar simbólico para los empresarios del Prodes Tamal- Lechona, y en general para los habitantes de la ciudad Ibagué, es la plaza de mercado, dado que es un punto de referencia tradicional para acceder a la comida típica de dicha región, el tamal y la lechona. Por último, el Cluster Textil Confección del Tolima es un símbolo de desarrollo asociativo para la ciudad de Ibagué, y específicamente para el sector confección, ya que es el encargado de organizar y liderar la feria Ibagué Maquila y Moda. Estos símbolos representativos de algunos Prodes, son formas visibles de manifestar identificación cultural con el grupo de pertenencia. Esto mismo se presenta con los héroes y rituales, los cuales favorecen la unidad del grupo.

Algunos personajes o héroes reconocidos a nivel nacional por su aporte al desarrollo de la estrategia Prodes en Colombia son: Zoilo Pallares Villegas, Jairo Díaz Valencia, y Juan Alfredo Pinto. Son personas vivas, apreciadas por sus habilidades para liderar procesos asociativos en Colombia; aportaron las ideas, los conceptos y las metodologías para empezar a implementar en Colombia una estrategia de asociatividad focalizada principalmente hacia las Mipymes.

Los rituales también son prácticas culturales, son espacios de interacción colectiva, en los que se comparten ideas, proyectos y el quehacer propio de cada organización. Cuando los empresarios de un Prodes participan de una feria empresarial o comercial, están legitimando ante la sociedad y ante el medio empresarial su pertenencia al grupo asociativo. No todos los Prodes han participado ceremonialmente en ferias o eventos empresariales representativos de su sector, los únicos Prodes en los que se identificó participación en algunos eventos son: Pro-Bebé, Vía Primavera, Tamal-Lechona, y Confección. Los Prodes Ropa para Bebé y Vía Primavera ubicados en Antioquia, asiste anualmente a la feria nacional de Colombia Moda; el Prodes Vía Primavera promueve y desarrolla la feria Vía Primavera llevada a cabo en la región antioqueña. En la ciudad de Ibagué se desarrollan los siguientes eventos, así: la celebración anual del Día del Tamal en la que participa el Prodes Tamal-Lechona, la feria Ibagué Maquila y Moda en la que tienen cabida algunos empresarios del Prodes Confección, y la feria Expo-Tolima a la que se convoca cada uno de los empresarios de los Prodes creados en la región del Tolima. Para participar en estos eventos anuales los empresarios de los Prodes deben llegar a acuerdos colectivos relacionados con logística de participación y promoción del Prodes, y de las empresas que hacen parte de dichos grupos asociativos. 
Tabla 3. Prácticas: Símbolos, héroes y rituales en los Prodes objeto de estudio.

\begin{tabular}{|c|c|c|c|}
\hline Nueve Prodes de Colombia & Símbolos & Héroes & Rituales \\
\hline Común a todos los Prodes & $\begin{array}{l}\text { - Los Profos de Chile. } \\
\text { - Acopi. } \\
\text { - Acopi Antioquia. } \\
\text { - Los gansos. }\end{array}$ & $\begin{array}{l}\text { - Zoilo Pallares. } \\
\text { - Jairo Díaz } \\
\text { Valencia. } \\
\text { - Juan Alfredo } \\
\text { Pinto. }\end{array}$ & - Sin identificar. \\
\hline Metalmecánica Intec & - Sin identificar. & - Sin identificar. & - Sin identificar. \\
\hline Artes Gráficas & - Sin identificar. & - Sin identificar. & - Sin identificar. \\
\hline Pro-Bebé & - Sin identificar. & - Sin identificar. & - La Feria Colombia Moda. \\
\hline Energía & - Sin identificar. & - Sin identificar. & \\
\hline Vía Primavera & - Árbol Vía Primavera. & - Sin identificar. & $\begin{array}{l}\text { - La Feria Colombia Moda. } \\
\text { - Feria Vía Primavera. }\end{array}$ \\
\hline Tamal-Lechona & - Plazas de Mercado. & - Sin identificar. & $\begin{array}{l}\text { - Día del Tamal. } \\
\text { - Feria Expo - Tolima. }\end{array}$ \\
\hline Confección & $\begin{array}{l}\text { - Cluster Textil Con- } \\
\text { fección Diseño y } \\
\text { Moda. }\end{array}$ & - Sin identificar. & $\begin{array}{l}\text { - Feria Ibagué Maquila y } \\
\text { Moda. } \\
\text { - Feria Expo - Tolima. }\end{array}$ \\
\hline Tics & - Sin identificar. & - Sin identificar. & - Sin identificar. \\
\hline Metalmecánica (caldas) & - Sin identificar. & - Sin identificar. & - Sin identificar. \\
\hline
\end{tabular}

Fuente: Construcción propia, 2013.

\section{c. Valores centrales compartidos en los Prodes}

Los valores comunes identificados en los Prodes objeto de estudio fueron: confianza, compromiso, respeto, solidaridad, persistencia y autonomía. Los valores filosóficos indicados en la política interna y direccionamiento estratégico de la estrategia de asociatividad Prodes son: Autogestión, democracia, transparencia, respeto y solidaridad (Pallares, 1999). Al comparar estos valores centrales compartidos por los Prodes a través de prácticas históricas de interacción, con los valores filosóficos propuestos para el diseño de la estrategia de asociatividad, se encuentra que hay coincidencia en los valores de respeto y solidaridad.

Dentro de los valores centrales compartidos por los Prodes en estudio, aparece la confianza y el respeto en primer lugar, cinco Prodes los han interiorizado; seguidos de valores como el compromiso y la solidaridad, implícitos en cuatro de los Prodes objeto de la presente investigación; y la persistencia, identificada en tres grupos asociativos en los que se encuentran dos de los Prodes más antiguos, así: Metalmecánica Intec (9 años) y Artes Gráficas (5 años). La autonomía es un valor propuesto por Acopi y aceptado por cada uno de los empresarios de los Prodes estudiados. Este valor le da la plena seguridad al empresario que al participar en el grupo asociativo conserva la independencia y autonomía para gestionar su propia organización. La mayoría de Prodes estudiados en Antioquia tienen entre 
cinco y seis valores centrales compartidos por los empresarios de dichos grupos, a excepción del Prodes Energía que presenta tres valores; mientras en los Prodes estudiados en Ibagué, no hay valores centrales compartidos por los empresarios de los grupos, solo se práctica la autonomía, valor que ha sido sugerido por Acopi, y que es transversal a cada uno de los grupos Prodes en estudio. En el Prodes Tics, el único valor compartido que logra identificarse entre miembros del grupo es el respeto.

Tabla 4. Valores de los Prodes objeto de estudio

\begin{tabular}{|l|c|c|c|c|c|c|c|}
\hline $\begin{array}{c}\text { Nueve Prodes de } \\
\text { Colombia }\end{array}$ & $\begin{array}{c}\text { Confian- } \\
\text { za }\end{array}$ & $\begin{array}{c}\text { Compro- } \\
\text { miso }\end{array}$ & Respeto & $\begin{array}{c}\text { Solidari- } \\
\text { dad }\end{array}$ & $\begin{array}{c}\text { Persis- } \\
\text { tencia }\end{array}$ & $\begin{array}{c}\text { Autono- } \\
\text { mía }\end{array}$ & $\begin{array}{c}\text { No de valo- } \\
\text { res compar- } \\
\text { tidos por el } \\
\text { Prodes }\end{array}$ \\
\hline Metalmecánica Intec & $\mathrm{X}$ & $\mathrm{X}$ & - & $\mathrm{X}$ & $\mathrm{X}$ & $\mathrm{X}$ & 5 \\
\hline Artes Gráficas & $\mathrm{X}$ & $\mathrm{X}$ & $\mathrm{X}$ & $\mathrm{X}$ & $\mathrm{X}$ & $\mathrm{X}$ & 6 \\
\hline Pro-Bebé & $\mathrm{X}$ & $\mathrm{X}$ & $\mathrm{X}$ & $\mathrm{X}$ & - & $\mathrm{X}$ & 5 \\
\hline Energía & $\mathrm{X}$ & - & $\mathrm{X}$ & & - & $\mathrm{X}$ & 3 \\
\hline Vía Primavera & $\mathrm{X}$ & $\mathrm{X}$ & $\mathrm{X}$ & $\mathrm{X}$ & $\mathrm{X}$ & $\mathrm{X}$ & 6 \\
\hline Tamal -Lechona & - & - & - & - & - & $\mathrm{X}$ & 1 \\
\hline Confección & - & - & - & - & - & $\mathrm{X}$ & 1 \\
\hline Tics & - & - & $\mathrm{X}$ & - & - & $\mathrm{X}$ & 2 \\
\hline Metalmecánica (caldas) & - & - & - & - & - & - & - \\
\hline Total Prodes & 5 & 4 & 5 & 4 & 3 & 8 & 29 \\
\hline
\end{tabular}

Fuente: Construcción Propia, 2013.

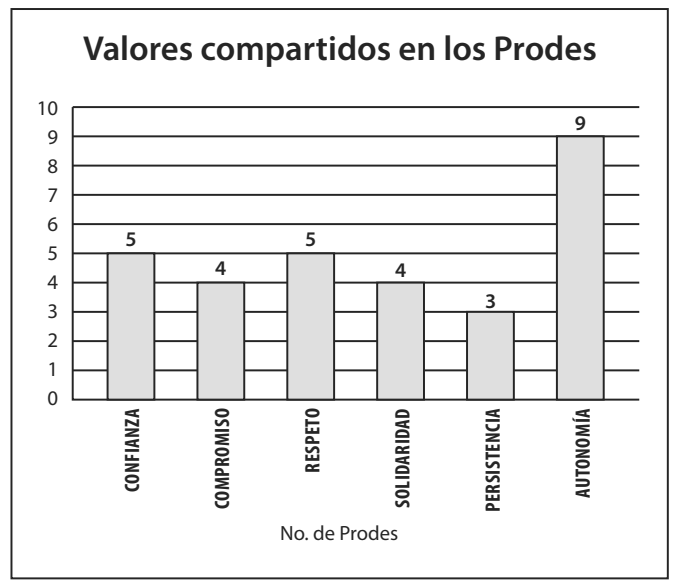

No. de Valores compartidos por Prodes

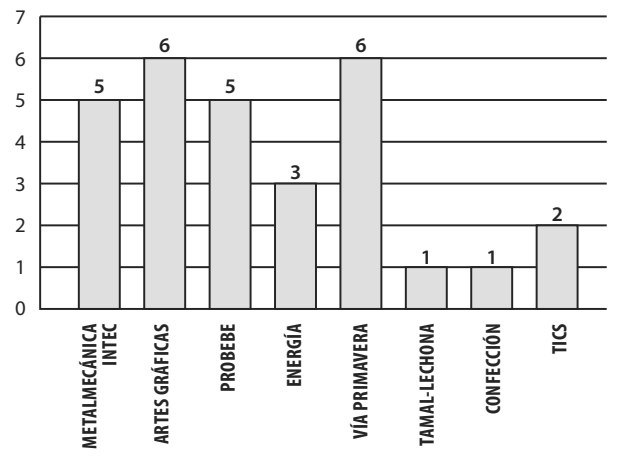

Fuente: Construcción Propia, 2013. Fuente: Construcción Propia, 2013. 


\section{CONCLUSIONES}

El presente artículo ha buscado identificar algunas manifestaciones de identificación cultural en los Prodes objeto de estudio, como es el caso de los Prodes Metalmecánica Intec, Artes Gráficas, Pro-Bebé, Energía, Vía Primavera, TamalLechona, Confección, y Tics. En el presente estudio, más que hacer alusión a un "cultura del Prodes", se hace alusión a manifestaciones de identificación cultural en dichos grupos asociativos, entendidas como mitos, estereotipos regionales, símbolos, héroes, ritos, prácticas y valores que hacen que un individuo u organización se sienta miembro o integrante de un grupo que adopta ideas, creencias y hábitos comunes. Para el análisis de dichas manifestaciones de identificación cultural se toma como base el modelo de Hofstede (1999), el cual está soportado por prácticas y valores compartidos por un grupo social, y que se construyen históricamente a través de procesos de interacción, comunicación y socialización entre individuos y organizaciones (Hofstede, 1999).

Las acciones de un Prodes pueden estar influenciadas por sus símbolos, héroes y rituales. En el surgimiento de los Prodes en Colombia han jugado un papel central, símbolos estratégicos como: los Profos de Chile, Acopi Nacional y Acopi Antioquia; personajes fundadores de los Prodes en Colombia que se identifican como "héroes" y creadores de dicha estrategia asociativa, así: Zoilo Pallares Villegas, Jairo Díaz Valencia y Juan Alfredo Pinto. No se identifican eventos, ferias o rituales que permitan congregar los Prodes existentes en Colombia; es decir, no hay un evento nacional de Prodes, o de experiencias Prodes en Colombia.

Tanto los símbolos como los héroes y los rituales tienen implícitos acciones y acuerdos colectivos que reflejan el grado de adscripción que tienen los individuos a un grupo social. En los Prodes los símbolos, héroes y rituales son manifestaciones de identificación y pertenencia a un grupo, independientemente si se han creado o no relaciones sociales fuertes entre integrantes del mismo. La consolidación de valores centrales compartidos en un Prodes, no de valores aceptados, parece ser una muestra que el grupo social ha invertido tiempo en el intercambio de experiencias, las cuales han permitido definir algunas prácticas, normas y valores compartidos, y que a la larga son manifestaciones de identificación cultural con el grupo de pertenencia. Los valores se crean porque hay una historia incorporada en las relaciones (Schein, 1985).

Colombia presenta diversidad de culturas, y se pueden encontrar algunos rasgos identitarios e identificarios propios de regiones como: Antioquia, Bogotá, Caldas y Tolima (regiones tenidas en cuenta para realizar la investigación), pero también es posible que en los Prodes regionales objeto de estudio se identifiquen características comunes en las empresas que lo integran, pero características culturales no exclusivamente relacionadas con la ubicación geográfica (regional), sino también rasgos culturales, relacionados con el nivel de "prácticas" propuesto por Hofstede (1999). La cultura se construye históricamente a través de las interacciones del individuo con diferentes grupos sociales (familia, escuela, empresa), y los Prodes tiene historias, prácticas, símbolos que los identifica, y al mismo tiempo los diferencia de otros Prodes ubicados en otras región y en sectores económicos diferentes. 


\section{REFERENCIAS}

1. ACOPI Presidencia Nacional. (2001). Programa de Desarrollo Empresarial Sectorial (PRODES): una estrategia asociativa para la competitividad de las empresas colombianas. "Cooperar para competir", pp. 1- 28.

2. ACOPI Seccional Tolima (2010). ¿Qué es Prodes?. (Online). En:<http://www.acopitolima.com/index. php?tip=cat\&id=9>. Consultada: Junio 21 de 2014.

3. AGUIRRE, A. y PINTO, M. (2006). Asociatividad, Capital Social y Redes Sociales. Revista Mad. Departamento de Antropología. Universidad de Chile. Vol. 15, pp. 74-92. URL:< http://www.revistamad.uchile. $\mathrm{cl} / 15 /$ aguirre.pdf $>$.

4. AGUIRRE, A.; Rodríguez, A. (1995). Patios abiertos y patios cerrados. Psicología cultural de las organizaciones. Barcelona: Editorial Boixareu Universitaria - Marcombo.

5. ANDRADE DE SOUZA, M. G. (2005). Prejuicio, estereotipo y discriminación: un análisis conceptual a partir del caso de la "aporofobia". Pontificia Universidad Católica de Río de Janeiro, Consejo Nacional de Desarrollo Científico y Tecnológico (CNPq). Brasil: Universidad de Valencia, pp. 1- 21. Online: <http:// www.nodo50.org/redrentabasica/descargas/gustavoandrade_valencia.Pdf >.

6. ANDREWS, S.; BASLER, C.; COLLER, X. (1997). Redes, cultura, e identidad en las organizaciones. Revista Reis. Vol. 2, pp. 31-56. ARCE, S. (2006). Asociatividad empresarial y competitividad. Miércoles del exportador. Bogotá D. C: Prompex, pp. 1-20.

7. ARCILA, E. M. (2006). El elogio de la dificultad como narrativa de la identidad regional en Antioquia. Revista Historia Crítica, No. 32, pp. 38-66.

8. COGOLLO, K. V.; LEÓN, L.; VERGARA, B. (2005). Cartagena de Indias y la asociatividad como estrategia competitiva de sobrevivencia en el desarrollo local. Artículo producto de la investigación: "Redes socio - políticas constituidas por el pequeño y mediano empresario cartagenero en el desarrollo local. Universidad de Cartagena, Facultad de Ciencias Sociales y Educación. Revista Palabra, № 6, pp. $28-41$.

9. CONGRESO DE COLOMBIA. Ley 590 de 2000.

10. DIMAGGIO, P. (1994). Culture and Economy. En: Smelser, N. \& Wedborg, R., The Handbook of Economic Sociology. Princeton: Princeton University Press.

11. EBERS, M. (1997). Explaining Inter-Organizational Network Formation. En: EBERS, M. The Formation of Interorganizational Networks, Oxford University Press, Nueva York, pp. 3-40.

12. EVERED, R. (1983). The Language of Organizations: The case of the Navy. En: Pondy, L.; Frost, P.; Morgan, G. \& Dandridge, T., Organizational Symbolism. Greenwich: JAI.

13. FERNÁNDEZ, P. A. (2011). Prejuicios y estereotipos: Refranes, chistes y acertijos, reproductores y transgresores. Revista de Antropología Experimental, Universidad de Jaén (España), № 11, 2011. Texto 22, pp. 317- 328.

14. FORSYTH, D. R. (2006). Group Dynamics. ( $4^{\text {th }}$ Ed)USA:Thompson Wadsworth. 
15. GELFAND, M.; EREZ, M. \& AYCAN, Z. (2007). Cross-cultural organizational behavior. Annual Review of Psychology. Vol. 58, N 1, pp. 479-514.

16. HERSKOVITS, M.J. (1955). Cultural Anthropology. New York: Knopf.

17. HOFSTEDE, G. (1983). The Cultural Relativity of Organizational Practices and Theories. Journal of International Business Studies, Vol. 14, No. 2, Special Issue on Cross- Cultural Management, (Autumn, 1983), pp. 75- 89 Published by: Palgrave Macmillan Journals Stable URL: <http://www.jstor.org/ stable/222593 Accessed: 21/04/2008 05:30>.

18. HOFSTEDE, G. (1999). Cultura y Organizaciones: El Software Mental, la Cooperación Internacional y su Importancia para la Supervivencia. Madrid: Alianza Editorial. pp. 1- 445.

19. HOUSE, R.; HANGES, P.; JAVIDAN, M.; DORFMAN, P.; GUPTA, V. (2004). Culture, leadership and Organizations, The Globe Study of 62 societies. London: Sage Publications Ltda.

20. IGUERA, M. (2006). Asociatividad en Pymes. Argentina. pp. 1 - 10. (Online). En: <http://www.lecantolas40.com.ar/egifts/asociatividad.pdf>. Consultada: Abril 18 de 2013.

21. KLUCKHOHN, C. (1954). Culture and behavior. En: Lindzey, G., Handbook of Social Psychology. Cambridge, MA: Addison-Wesley. Vol. 2, pp. 931-76.

22. LLANQUE, F. R. (2002). Redes sociales y cultura organizacional en entidades públicas. Revista de antropología experimental. Universidad de Jaén: Facultad de Humanidades y Ciencias de la Educación. $\mathrm{N}^{\circ} 2$, pp.1578- 4282 .

23. MACKIE, M. M. (1973). Arriving at truth by definition: Case of stereotype inaccuracy. Social Problems, Vol. 20, pp. 431-447.

24. MIDEPLAN (2002). Estrategias de Intervención Integral a favor de Familias de Extrema Pobreza. Santiago, Chile: MIDEPLAN, pp. 1- 64.

25. MINISTERIO DE COMERCIO, INDUSTRIA Y TURISMO. MCIT (2005). Cadenas, Redes Empresariales y Asociatividad. (Online). En: <http://www.mincomercio.gov.co/econtent/documentos/competitividad/2006/CadenasRedesAsociatividad.pdf>. Consultada: Mayo 12 de 2013.

26. OGLIASTRI, E.; MCMILLEN, C.; ALTSCHUL, C.; ARIAS, M. E., BUSTAMANTE, C.; DE, DÁVILA, C.; DORFMAN, P.; DE LA COLETTA, M. F.; FIMMEN, C.; ICKIS, J., \& MARTÍNEZ, S. (1999).Cultura y liderazgo organizacional en diez países de América Latina. El estudio Globe. Academia. Revista Latinoamericana de Administración. Vol. 22, pp. 29-57.

27. ORTIZ C., F. (2002). Programa de Desarrollo Empresarial Sectorial - PRODES: Estrategia asociativa para la pyme colombiana. Forjadores de Paz y Progreso. ACOPI Presidencia Nacional, pp. 1- 27.

28. OTT, J.S. (1989). The Organizational Culture Perspective. Pacific Grove: Brooks/Cole.

29. PALLARES VILLEGAS, Z. (1999). La asociatividad empresarial: Una respuesta de los pequeños productores a la internacionalización de las economías. Director nacional PRODES. Bogotá, pp. 1-9.

30. PALLARES VILLEGAS, Z. (2004). Asociatividad empresarial estrategia para la competitividad. Bogotá D.C. (Colombia): Fondo Editorial Nueva Empresa, pp. 1-319.

31. POWELL, W. W. (1990). Neither Market Nor Hierarchy: Network Forms of Organization. En: Staw, B. \& Cummings, L. L. Research in Organizational Behavior, Greenwich, CT: JAI Press, pp. 295-336. 
32. ROSALES, R. (1997). La asociatividad como estrategia de fortalecimiento de las Pymes. Capítulos, 7 y 9. № 51. Caracas (Venezuela): Sela.

33. SCHEIN, E. H. (1996). Culture: The Missing Concept in Organizational Studies. Administrative Science Quarterly. Vol. 41, pp. 229-240.

34. Schein, E. H. (1985). Organizational Culture and Leadership, San Francisco: Jossey-Bass.

35. SHWEDER, R. \& LEVINE, R. (1984). Culture Theory: Essays on Mind, Self, and Emotion. London: Cambridge University Press.

36. SKINNER, B.F. (1981). Selection by consequences. Science. Vol. 213, pp. 501- 504.

37. TRIANDIS, H. C. (1972). The Analysis of Subjective Culture. New York: Wiley.

38. TRIANDIS, H. C. (1994). Culture and Social Behavior. New York: McGraw- Hill.

39. UZZI, B. (1997). Social structure and competition in interfirm networks: The paradox of embeddedness. Administrative Science Quarterly. Vol. 42, N 1, pp. 35-67.

40. VEGA, R. J. (2008). Asociatividad. Consorcio Asecal Mercurio Consultores. Perú: Proyecto de Cooperación UE-PERU / PENX, pp. 1- 38.

41. VINACKE, W. E. (1956). Stereotypes as social concepts. Journal of Social Psychology. Vol. 46, pp. 229243.

42. WILKINS, A.L. \& Ouchi, W.G. (1983). Efficient Cultures: Exploring the Relationship between Culture and Organizational Performance. Administrative Science Quarterly. Vol. 28, pp. 468-481. 